\title{
V.
}

\section{Einige Erläuterungen}

zu Hrn Prof. Hanfleen's neuer magnetifchen'Neigungs-Karte auf beiliegenden $K_{P} \int t f \ln I I I u_{\text {. IV }}$;

$$
\text { von Ginвет T. }
$$

Die magnetifche Neigungs - Karte für das J. 1780 auf Tafel IV, ift eine verbeflerte Ausgabe der in Hrn Hanfteen's "Magnetismus der Erde"s auf Taf. VII enthaltenen Neigungs-Karte. Sie reicht, wie diefe, nach Norden nur bis zu der Linie hinauf, in welcher die Neigung $80^{\circ}$ beträgt. Erft durch die Entdeckungs-Reifen unter Rofs und Parry nach dem nord weftlichen Polarmeere, in den Jahren 1818 bis 1820 , alfo nachdem $\mathrm{H}_{\mathrm{rn}}$ Hanfteen's Werk über den Magnetismus der Erde fchon erfchienen war, haben wir hinlanglich viele und hinlänglich genaue Beobachtungen erhalten, um die Lage des Punktes, wo die Neigung $9^{\circ}$ ift, mit einiger Zuverlâfrgkeit nachzuweifen, und die Linien gleicher Neigung von $80^{\circ}$ bis $9^{\circ}$ Inclinåtion von Grad zu Grad zu zeichnen. Hr. Hanfteen hat in dem vorftehenden Auffatze felbft umftundliche Rechenfchaft von leiner Berechnung diefer neuen, zur Kenntnifs des Erd-Magnetismus befonders wichtigen Beobachtungen und deren Ergebnifen, 'und ron der Art gegeben, wie er fie anf dem hier auf Tafel III zum erften Male erlcheinenden, und nach cinem gröfsern Maafs- 


\section{$\left[\begin{array}{ll}29^{3} & ]\end{array}\right.$}

liabe als die Karte verzeichneten - Erganzungs - Blatte dargeftellt hat. Es bedarf daher ciefe Ergänzungs Karte keiner weiteren Eirklärung. Doch wird es gut ley'n, noch ein Mal darauf aufunerkfam 'zu machen, dals fie anch die jaut den beiden Entdecknngs - Reifen beobachteten Neigungen in Zallen darftellt (die kleinen Kreife bezeichnen die Stellen der Beobachtung), und dals fich auf ilır auch einige der merk würdigften $\mathbf{A b}$ weicluungen der Nordfpitze der Magnetnadel angegeben linden, durch kleine Pfeile, welche von dem Orte der Beobachtung ausgehn und durch ilure Lage die Richtung des magnetilchen Meridians dalelbft darftellen. Da wo fich an der Oberfläche der Eıde lotlıreclite Ebneı durch diefe Pfeile gelegt durchkreuzen, ift der nördliclie Convergenz - Punkt der magnetifchen Abweichungen, und man überfielıt daher lier die Lage diefes Punktes zu Folge der Beobachtungen rechit gut. Dafs iln nicht alle übereinfimmend und mit dem Punkte, wo die Neigung $90^{\circ}$ ift, zufammenfallend geben, liegt unftreilig nur an der Unvollkommenheit der Alweichungs-Beobaclitungen in einer Gegend, wo die die Magnetnadel in horizontaler Ebne richtende Kraft dem Verfcliwinden nahe ift.

Folgende Erläulerungeu der verbefTerten NeigungsKarte felbft, (Taf. IV), entlelnne ich theils ans dem zweiten Hauptfïcke voir Hrn Hanfteen's ,Magnetismus der Erde", welches von den Neigungs-Linien liandelt, theils ans einem Schrciben dellelben an micle in dem diefsjälır. Jnnuarftück m. Annalen.

Erft feit dem J. 1576 ift dic Neigung der Magnetnadel bekaint ; ein nathematifcher Inftrumentmacher in London, Norman, nalim fie zuerft walır. Schon Hudfon, 


\section{[ $\quad \begin{array}{ll}295 & 3\end{array}$}

der Cook feiner Zeit, ftellte auf feimen drei Entdokkungsreifen zur Auffindung einer nordweftlichen oder nordöftichen Durchifahrt, gute Neigungs-Beobachtungen an; fie liaben $\mathrm{Hrn}$ Hanfteen in den Stand gefetzt ein Neigungskärtchen für das J. 1600 zu entwerfen, das von $75^{\circ}$ bis $87 \frac{1}{2}^{\circ}$ nördl. Neigung gelıt. Die Linien gleicher Neigung ftellte zuerft Wilke in einer Karto dar, in den Schriften der Schwed. Akad. der Wiffenfch. anf 1768 , nach Beobachtungen Cunnigham's, Feuillée's, La Caille's und des Schiffskapitäns Eckeberg; Hr. Hanfteen giebt fie mit einigen Verbelferungen and zweckmâfsig verkleinert, als Neigungskarte für das J. 1700. Ans ihr folgert er, dafs, da die Linie ohne Neignng, oder der magnetifche Aequator, ihre gröfste lüdliche und nördliche breite in den Meridianen, jene von Ferro mit $14^{\circ}$, diele von Japan mit $29^{\frac{1}{2}} 0$ liabe, fio kein gröfster Kreis fey; und dals die Neigung um den magnetifichen Aequator fich doppelt fo f'clinell, iiber $70^{\circ}$ Neigung hinaus aber nur halb fo fchnell, als die geographifche Breite verändere; ein Satz, den er in feinem Werke aus der Theorie des Magneten lelbft zu bewahren gefuclit hat.

Scine eigne Karte der Linien gleicher Neigung für das J. 178o, gründete $\mathrm{Hr}$. Hanfteen vorzüglich auf die Beobachtungen der magnetifchen Neigung, welche auf der zweiten und dritten Reife Cook's und auf der Entdeckungsreife I.a Peyroufe's angeftellt worden waren, und auf dic möglichft genaue Beftimmung der Lage des magnetifichen Aequators diefen und andern neuen Bpobachtungen gemäls. Er bedionte fich hierbei fchlın eines ganz ahnlichen Verfahrens, als das, welclres $\mathrm{Hr}_{r}$. Biot in Ceinem Berichte über Hrn Mor- 


\section{[ 394 ]}

let's Belfimmung des magnetifchen Aequators, dệ der Lefer im diefojuhr. Januarftücke meiner Annalen gefunden lıat, als eines finnreichen diefem franzöfifchon Gelehrten angehörenden Interpolations - Verfahrens rühmt, um aus Beobachtungen an Orten, wo die Neigung gering ift, die Lage von Punkten im magnetil'chen Aequator zu finden, nur dafs ihm hierzu ein anderer Satz dient. Nämlich, dafo wenn in einer Breite (B) eine geringe magnetifcheNeigung $(\mathrm{N})$ beobachtet worden iff, dort in der Breite $=\mathrm{B}-\frac{1}{2} \mathrm{~N}$ die Neigung $=0$ fey, und alfo ein Punkt des magnetifchen Aequators liege. Durch folche Interpolationen und genaue Discuffionen von Beobachtungen Eckeberg's, Abercrombie's, Le Gentil's, La Peroufe's, Paton's, Krufenftern's und andre (S. 47 bis 60 feines Werks) erhielt $\mathrm{Hr}$. Hanfteen 70 Beftimmungen von Punkten in der Linio olune Neigung, nach Länge und Breite, welcho er S. 60 in einer Tafel zufammenftellt. Ihnen gemäfs verzeichnete er den magnetifchen Aequator auf feiner Neigungs-Karte für 1780, auf Taf. VII des Atlalles zu feinem "Magnetisnus der Erde." "Es wäre von Wichtigkeit, bemerkt Hr. Hanfteen S. 62, die Richtung der übrigen Neigungslinien eben fo ausführlich zu beweifen, um diefer Karte diejenige Zuverlalligkeit zu ertheilen, welche erforderlich ift, wenn fie zur Grundlage einer theoretifchen Unterfichung des Erdmagnetismus dienen foll; aber es würde diefes zu weitlăufig und zu ermüdend feyn. Es genüge alfo dio Verficlıerung, dafs in Rückficht der übrigen Neigungslinien dielelbu Genauigkeit beobachtet worden ift."

Dafo dio Linie ohne Neigung kein gröfoter Kreis ift, "welchos Untiand allein fchon cino unüberwind- 


\section{$\left[\begin{array}{lll}295 & \end{array}\right]$}

liche 8chiwierigkeit für jeden ift, welcher der Erde nur Eine Magnetaxe oinräumt ", boftutigte fich aus diefer Darftellung vollkommen. Nimmt man alle Längen weftlich von Greenwioh, fo durchlclnneidet, dieler Karte zn Folge, der magnetifche Aequalor den aftronomifchen im Südmeere in $110^{\circ}$ und in Afrika in $235^{\circ}$ Lange, dort unter einem Winkel von ungefahr $7 \frac{x}{2}$, hier von $21_{2}^{\frac{1}{2}}$, und er erreicht die gröfste füdliche

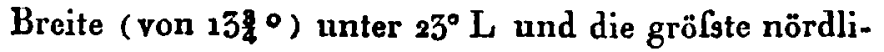
che Breite (von $115^{\circ}$ ) unter $295 \mathrm{~L}$, welcho beide $\mathrm{Me}$ ridiane nur um $88^{\circ}$ von einander ablitehn.

Warum diefo Beftimmung die Walırheit noch nicht ganz traf, davon giebt Hr. Prof. Hanfteen den Grund in den Briefen an, welche in diefojälır. Jannarftück S. 23 n, 120 m. Annal. ftehn. Er hatte den Band "aftronomifclier Beobachtungen auf der Entdeckungsreife von 1776 bis 1780 in das nördliche Stille Meer unter Kapit. Cook und Lieuten. King " niclıt benutzen können, erhielt jhn erft im J. 1820, und überzengte fich aus den in demlelben mitgetheilten NeigungsBeobachtungen, dafs er Unrecht gehab: habe „irre gelcitet durch fellerhafte Beobachtungen La Peroufe's und Krufenftern's " der auf ihnen gegrüudeten Behauptung des Hrn Biot zu widerfprechen, die Linie ohne Neigung Schneide den Erd-Aequator iṇ vier Punkten [oder komme vielmehr mit dem Aequator an vier verfchiednen Stellen zufammen, wie Hr. Biot diefe Behauptung nach Hru Morlet's Arbeit verändert hat ] "). „Ich labbe (heifst es im erftern Briefe an Hrn Akad. v. Yclin) in Anfehung der Linion in Stillen Meere ei-

•) Vorgl. d. diefsjähr. Januarttück S. 19. G. 


\section{[ 296 ]}

nen Fehler begangen, indem mir Cook's und Bayly's Original-Beobachtungen auf der dritten Keife abgingen. Meine Karte giebt nur zwei folche Durch fchnittspunkte. Allein in der Folge erfalt ich, dafs jenen Beobaclitungen zu Folge, die Linie ohne Neigung in der That den Aequator an vier Stellen folıneidet, nänlich in $108^{\circ}, 125^{\circ}, 170^{\circ}, 235^{\circ}$ weftl. L. von Greenwich. "* Und in dem zweiten Briefe l'agt Hr. Hanfteen über leine nach jenon Beobachtungen verbeferte Neigungskarte, welche lier anf Taf.IV erfcheint: „Im lïdliclsen Theile des Stillen Meeres bedurften die Linien gleicher Neigung keiner Verändernug; aber etwa von $10^{\circ}$ f. Br. an bis zur Belirings - Strafse haben fie eine Biegung gegen Süden erhalten, welche einen noch gröIsern Parallelismus im ganzen Syfteme hervorbringt, und einen nenen Beweis für zwei Magnetaxen der Erde giebt. Ich betrachte nunnelir diefe Karte als fo genau, dafs ich jeden auffordern darf, eine Beobachtung um 1780 vorzuweifen, die nicht beweislich falfch wäre, wemn fie um melır als 1 oder $1 \frac{x}{2}^{\circ}$ von der Karte abweicht. Die Karte ift fogar genaner als jede einzelne Beobachtung; denn fie bietet die Mittelzahlen der ganzen Totalfumme der Beobachtumgen dar, worin folglich alle Beobachtungs - und Oertlichkeits - Fehler verfchwinden."

Ift ein folches Vertrauen in die Zuverluffigkeit diefer Karte vielleicht aucis viel zu grol's, fchon wegen der mangelhaften Belchaffenlıeit der mehrften Neigungs-Beobachtungen, auf welchen fie beruht "), und des

") Von der bewundernswürdigen Menge genaller NeigungsBeobachtungen, weiche Kapitän Frejcinet von feiner Entdek. 


\section{[ $\quad 397$ ]}

völligen Mangels an Beobachtungen an manchen Gegenden der Erde, - lo erfüllt diefe Karte doch darin fchon jetzt ihren Zweck völlig, dafs fie, befonders in Vereinigung mit dem Erganzungs-Blatt anf Taf. III, eine zuverläfige, lediglich ans der Erfahrung entlehnte Grundlage für Theorien der magnetifchen Neigung abgiebt.

Zum Schlufe diefer Erläuterungen noch folgen. des Refultat, welchies Hr. Hanfteen aus der Vergleichung feiner Neigungs-Karten für 1600, für 1700 und für 1780 zieht: "Die nördliche Neigung nimmt in Nord-Amerika $z u$, in Europa $a b^{*}$ ), in öftlichen Afien und bei Japan au. Die lïdlicho Neigung nimmt bei Sïd-Amerika $a b$, ift um das Vorgebirge der guten Hoffnung beftändig, und nimmt bei den Sunda-Infeln und bei Neu-Holland ab."

kungsreife mitgebracht hat, fjebe das diefsjähr. Januarftlick $m$. Annalen S. 84 f. Gilb.

) Die alteften Neigungs - Beobachtungen in London find, Hrn Hanfteen zu Folge, um mehrere Grade zu klein, welches der Grund fey, warum von 1576 bis 1720 die Neigung in London zuzunehmen und feitdem erft abzunehmen fohien. Diefes glaubt er durch Vergleichung derfelben mit gleichzeitig zu Paris angeftellten Beobaclitungen bewăhren zu kónnen, indem Richer die Neigung in Paris im J. $167175^{\circ}$, Bond aber fie zu London im J. 1676 aur $73 \frac{1}{2}^{\circ}$. Norman im J. 1576 felbat nur $71^{\circ} 50^{\prime}$ fand.

$G$. 